\title{
Procedure Location
}

National Cancer Institute

\section{Source}

National Cancer Institute. Procedure Location. NCI Thesaurus. Code C117525.

The site in or on the body at which the procedure is performed. 